\title{
POLINSAR EXPERIMENTS OF MULTI-MODE X-BAND DATA OVER SOUTH AREA OF CHINA
}

\author{
Lijun Lu, Qin Yan, MinyanDuan, Yanmei Zhang \\ Chinese Academy of Surveying and Mapping, Lianhuachi west road, No 28,100830,Beijing, P.R.China \\ lulijun2002@gmail.com
}

\section{Commission VII, VII /7}

KEY WORDS: PolInSAR, land classification, objects detection, DSM mapping

\begin{abstract}
:
This paper makes the polarimetric and polarimetric interferometric synthetic aperture radar (PolInSAR) experiments with the highresolution X-band data acquired by Multi-mode airborne SAR system over an area around Linshui, south of China containing tropic vegetation and urban areas. Polarimetric analysis for typical tropic vegetations and man-made objects are presented, some polarimetric descriptors sensitive to vegetations and man-made objects are selected. Then, the PolInSAR information contained in the data is investigated, considering characteristics of the Multi-mode-XSAR dataset, a dual-baseline polarimetric interferometry method is proposed in this paper. The method both guarantees the high coherence on fully polarimetric data and combines the benefits of short and long baseline that helpful to the phase unwrapping and height sensitivity promotion. PolInSAR experiment results displayed demonstrates Multi-mode-XSAR datasets have intuitive capabilities for amount of application of land classification, objects detection and DSM mapping.
\end{abstract}

\section{INTRODUCTION}

Nowadays, Polarimetric and Interferometric Synthetic Aperture Radar (PolInSAR), as good combination the advantages of SAR Interferometry and SAR Polarimetry, has been widely applied to classification of crops and forests, man-made target detection, measurement of soil, quantitative tree height determination, ground topography mapping and etc. Different from the previous airborne PolInSAR system, Multi-mode-XSAR system developed by the China Electronics Technology Group Corporation 38 department (CET38) provide the possibility to simultaneously observe the ground surface more than once by altering transmitting and receiving mode of pulse signal. Therefore, it is essential to start exploring the potential of multimode-XSAR datasets for objects interpretation and digital surface model (DSM) mapping.

Section 2 is dedicated to the presentation of multi-mode-XSAR, the experimental airborne SAR system developed by CET38. And the multi-mode-XSAR dataset and the corresponding ground measurements are described. In Section 3 describes a polarimetric analysis for typical vegetations and man-made objects, some polarimetric descriptors sensitive to vegetations and man-made objects are selected for land classification and objects detection.

Then, section 4 concerns the phase information in the datasets. Considering characteristics of the multi-mode-XSAR dataset, a dual-baseline polarimetric interferometry method is proposed in this paper. On one hand, it guarantees the high coherence on fully polarimetric data; on the other hand, it combines the benefits of short and long baseline that helpful to the phase unwrapping and height sensitivity promotion.

Next, section 5 of this paper displays the PolInSAR experiment results of the land classification, man-made objects detection and DSM mapping. Finally, section 6 gives the conclusion of polInSAR experiment with multi-mode-XSAR data.

\section{MULTI-MODE-XSARSYSTEM AND DATASETS}

\subsection{Multi-mode-XSAR System}

Multi-mode-XSAR system is developed by CET38. The radar system is placed on China "Y-7" airborne platform and the mean flight velocity is nearly $120 \mathrm{~m} / \mathrm{sec}$, at $5000 \mathrm{~m}$ altitude. The radar incidence angle could be adjusted from 37 degree to 45 degree and the resolutions are $0.1 \mathrm{~m}, 0.4 \mathrm{~m}$ (azimuth resolution and slant resolution). Especially, it can provide three kinds of working modes including bistatic, ping-pang and mixed modes. The bistatic mode represents one antenna transmit signal and two antennas synchronously receive signals; the ping-pang mode represents antenna pairs alternately transmit and receive signal via the way of transferring transmit/receive(T/R)switch; the mixed mode represents the bistatic mode and the ping-pang mode alternately works at the cost of reducing system bandwidth. The table 1 gives the detailed configuration parameters of Multi-mode-XSAR system.

Table 1.Parameters of Multi-mode-XSAR system.

\begin{tabular}{|l|c|c|c|}
\hline \multicolumn{1}{|c|}{ Mode } & $\begin{array}{c}\text { Bandwidth } \\
{[\mathrm{MHz}]}\end{array}$ & Polar & $\begin{array}{c}\text { Swath width } \\
(\mathrm{km})\end{array}$ \\
\hline bistatic & 360 & Full & 2 \\
\hline ping-pang & 300 & Full & 2 \\
\hline mixed & 250 & Full & 1 \\
\hline
\end{tabular}

\subsection{Dataset and ground measurement}

CET 38 performed several flight plans in LingShui of the south of China from 2009 to 2010, acquiring about $2000 \mathrm{~km}^{2}$ PolInSAR datasets over LingShui area. At the same time, three trihedral and dihedral corner reflectors were deployed on the scene for polarimetric and geometric calibration. The three trihedral corner reflectors mostly centralized on the airfield were employed for polarimetric calibration, and i.e. are used to accurately register the different polarization images and to compute the differential delays between channels. The other three trihedral and dihedral corner reflectors widely spread all places are employed for geometric calibration, and i.e. are used to accurately correct geometric position. 


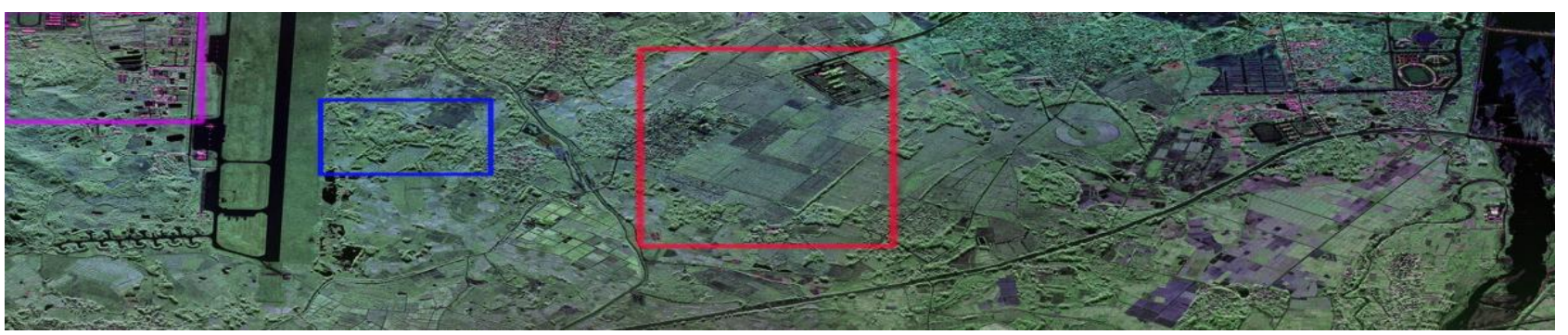

Figure.1.The colors ynthetized map of three polarizations basis (HH, HV, VV) of the LingShui area. The three test areas represented as red, blue and fuchsine color rectangles respectively.

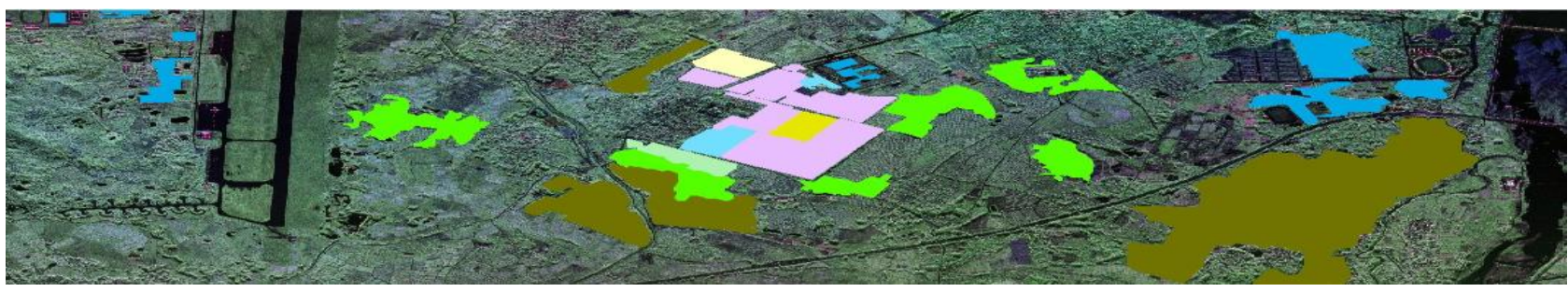

bare crop forest building areca lychee pawpaw mango

Figure.2.Ground measurements superimposed on the polarimetric color synthetized map.

During the period of data acquisition, the associated ground survey campaign was also implemented in test area, composed of bare surface, orchards, cropland, forests, and residential area, as shown in figure.2. Figure.1. presents the study area as a color composite where the backscattered powers corresponding to the three standard basis' polarizations ( $\mathrm{HH}, \mathrm{HV}, \mathrm{VV})$ are respectively coded in red, green, and blue. Orchards and forests appear in green color, buildings in red color, and cropland in blue or green color. The typical test areas marked with rectangles are selected as experimental areas of land classification, man-made objects detection and DSM mapping shown in figure. 2 . The test area1 in red color is selected as land classification experiment, test area 2 in fuchsine color is selected as man-made objects detection experiment, test area3 in blue color is selected as DSM mapping experiment.

\section{POLARIMETRIC ANALYSIS}

\subsection{Polarimetric Parameters}

The classical polarimetric parameters, the entropy, the alpha angle, and the anisotropy describe the polarimetric properties of different surfaces which have been proposed in (S.R.Cloude, 1997). As the alternative parameters, surface scattering fraction, scattering diversity and etc. can be used by a fairly simple algorithm (E.Colin, 2010). The alternative parameters will provide the easier way of image interpretation, classification and visualization. Therefore, as the new adjustment for polarimetric parameters, the alpha angle is replaced by surface scattering fraction, entropy is adjusted to the simpler formula avoiding any eigenvalue calculation(J.Praks, 2009), anisotropy retains the classical formula, scattering diversity is appended as the new polarimetric parameter.

\subsection{Polarimetric Analysis for Datasets}

Surface scattering fraction, entropy, anisotropy and scattering diversity of datasets are firstly computed over a $9 \times 9$ sliding window. To proceed to a more quantitative analysis of polarimetric parameters, some representative areas are analyzed

in surface scattering fraction /entropy, the surface scattering fraction /anisotropy and surface scattering fraction /scattering diversity planes with a $30 \times 30$ pixel window. Entropy-Surface fraction scatter plots presented in figure. 3 show that surface scattering fraction can discriminate between bare surface, forest, building and cultivated lands (crops and orchards). However, for different types orchards and crops, surface scattering fraction can not discriminate them. Entropy represents the best discrimination capability, as shown in figure.3, except for the same capability as surface scattering fraction has, it still can identify areca from other cultivated lands since it behaviours similar property of higher entropy with forest covered with more leaves. Figure.4. show that scattering diversity gives the results close to the entropy, but it represents the lower discrimination capability than Entropy. Figure.5. show that anisotropy can identify buildings from the other types well as the observations of eigenvalues are different. Therefore, surface scattering fraction, entropy, anisotropy are selected for land classification and objects detection as the following experiments.

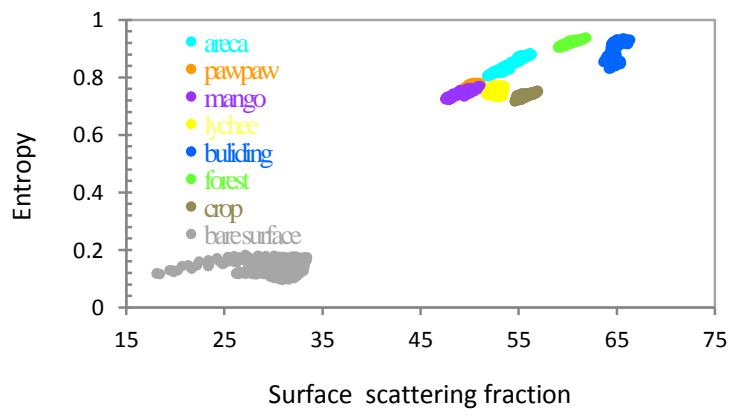

Figure.3.Entropy-Surface fraction scatter plot of different types of lands 


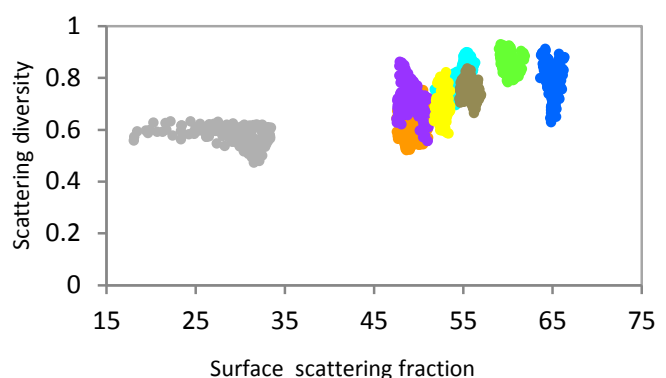

Figure.4. Scattering diversity-Surface fraction scatter plot of different types of lands.

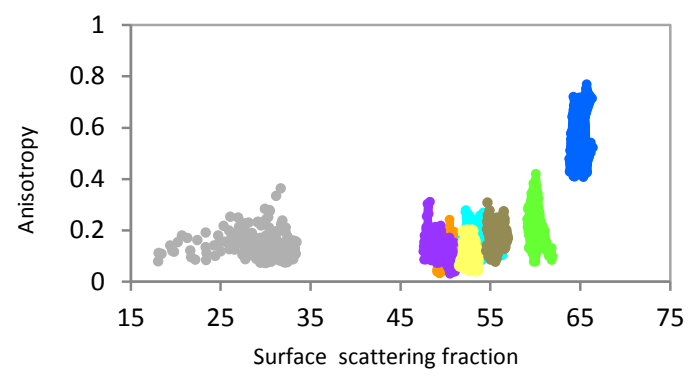

Figure.5. Anisotropy-Surface fraction scatter plot of different types of lands.

\section{PHASE ANALYSIS}

Multi-mode-XSAR system provides us the possibility of Multibaseline polarimetric interferometry research. In the case, dual baseline configuration can be achieved via mixed work mode of airborne SAR system.

A dual-baseline polarimetric interferometry method of combined characteristics of polarimetry and interferometry is proposed as follows.

\subsection{Coherence Optimization}

Fully polarimetric monostatic data can be represented inthe Pauli basis, assuming reciprocity, for one track, by the scattering vector $\mathrm{k}$, i.e.,

$$
K=\frac{1}{\sqrt{2}}\left(S_{H H}+S_{V V} \quad S_{H H}-S_{V V} \quad 2 S_{H V}+S_{V H}\right)^{T}
$$

Using the outer product formed from the scattering vectors and for images 1 and 2, we can define a $6 \times 6$ Hermitian,

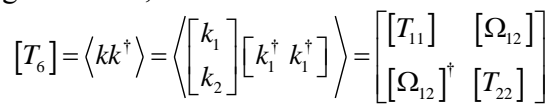

Where $<>$ represents the multi-looking operator, and $\dagger$ the Hermitian transformation. $\left[\mathrm{T}_{11}\right]$ and $\left[\mathrm{T}_{22}\right]$ represent the standard Hermitian coherency matrices that contain the full polarimetric information for each separate image. $\left[\Omega_{22}\right]$ is a new $3 \times 3$ complex matrix that containsnot only polarimetric information, but also the interferometric phase relations of the different polarimetric channels between both images.

According to the configuration Multi-mode-XSAR system, the temporal separations of acquisition time of different mode datasets are relative small and assume that scatter mechanisms (SMs) are similar at different mode of datasets. In (E.Colin, 2006) an Equal Scattering Mechanisms (ESM) optimization method is presented for single-baselines, which constrains the optimized SMs to be equal at the baseline end. It is based on the numerical range, properties of the modified polarimetric interferometric coherency matrix $\Pi_{12}$,

$$
\Pi_{12}=T_{e}^{-1 / 2} \Omega_{12} T_{e}^{-1 / 2} \text { where } T_{e}=\left(T_{11}+T_{22}\right) / 2
$$

The numerical range of matrix $\Pi_{12}, W\left(\Pi_{12}\right)$, can be seen as the the coherence $\tilde{\gamma}$ of $\Pi_{12}$.

$$
\begin{array}{r}
\tilde{\gamma}=w^{\dagger} \Pi_{12} w \text { where } w=\frac{\sqrt{T_{e}} \omega}{\omega^{\dagger} \sqrt{T_{e}} \omega} \\
W=\left\{x^{\dagger} \Pi_{12} x: x \in \mathbb{C}^{3}, x^{\dagger} x=1\right\}
\end{array}
$$

For the ESM case, complex unitary vectors $w_{1}, w_{2}$ corresponding to the SMs vectors are referred to the same SMs: $w_{1}=w_{2}=w$. The maximal coherence modulus of $\Pi_{12}$ corresponds to the numerical radius $\gamma\left(\Pi_{12}\right)$.

$$
r\left(\Pi_{1,2}\right)=\max \left\{\left|x^{\dagger} \Pi_{1,2} x\right|: x \in \mathbb{C}^{3}, x^{\dagger} x=1\right\}
$$

In (E.Colin, 2006) an iterative method is used to compute $\gamma$ $\left(\Pi_{12}\right)$ for the single-baseline case.

Mixed work mode can afford three baselines interferometric pairs, three interferograms with the optimized coherence are respectively formed according to the ESM method.

\subsection{Phase Estimation}

MLE (Maximum Likelihood Estimation) for Multi-baseline InSAR height estimation is thought as a good tool (S. Sauer, 2007). In the configuration of Multi-mode-XSAR system, mixed work mode provides two kinds of baseline pairs: one long baseline pair and two short baseline pairs, and length of long baseline pair is exactly two times as short baseline pair. The MLE method for three baseline pairs can be outlined as follows.

The received signal vector from the same point on the ground by three acquisitions can be expressed as

$$
\vec{V}^{(w)}=\left[V_{1}^{(w)}, V_{2}^{(w)}, V_{3}^{(w)}\right]^{T}
$$

$\mathrm{w}$ is the number of multi-looking.

Conditional probability density function $f$ corresponds to the interferometric phase

$$
f\left(\vec{V}^{(1)}, \vec{V}^{(2)}, \ldots \ldots, \vec{V}^{\left(N_{L}\right)} \mid \varphi\right)=\prod_{w=1}^{N_{L}} \frac{1}{\pi^{3}|\Gamma|} \exp \left(-\vec{V}^{(w)^{\dagger}} \Gamma^{-1} \vec{V}^{(w)}\right) \text { (8) }
$$

$\Gamma$ represents is Hermitian covariance matrices, diagonal elements are unitary, the off-diagonal elements are defined by

$$
\rho_{i, j}=\exp \left\{i \phi x_{i, j}\right\} \rho_{\text {noise }} \rho_{\text {spatio }} \rho_{\text {tempal }}
$$

$P_{i, j}$ is the correlation coefficient between the two acquired SAR images and it can be calculated via formula(6), $x_{\mathrm{i}, \mathrm{j}}$ is the ratio between baseline length of image $i$ and image $j$ and baseline length of image 1 and image $3,(i, j)=(1,3),(1,2),(2,3), x_{i, j}=1,1-p$, p.

Maximum log-likelihood function can be derived via logarithm operation of (8)

$$
\begin{aligned}
\max _{\varphi \in[-2 \pi, 2 \pi]} & \left\{\left(\left|\rho_{12}\right|-\left|\rho_{23}\right|\left|\rho_{13}\right|\right) \operatorname{Re}\left[\exp (-j \varphi(1-p)) \sum_{w=1}^{N_{L}} V_{1}^{\dagger(w)} V_{2}^{(w)}\right]\right. \\
& +\left(\left|\rho_{13}\right|-\left|\rho_{12}\right|\left|\rho_{23}\right|\right) \operatorname{Re}\left[\exp (-j \varphi) \sum_{w=1}^{N_{L}} V_{1}^{\dagger(w)} V_{3}^{(w)}\right] \\
& \left.+\left(\left|\rho_{23}\right|-\left|\rho_{12}\right|\left|\rho_{13}\right|\right) \operatorname{Re}\left[\exp (-j \varphi p) \sum_{w=1}^{N_{L}} V_{2}^{\dagger(w)} V_{3}^{(w)}\right]\right\}
\end{aligned}
$$

The phase $\varphi$ can be calculated in terms of expression (10), one key point is emphasized that the phase is extended from $[-\pi, \pi]$ to $[-2 \pi, 2 \pi]$ since the ratio of short and long baseline is exactly two. 
Dual baseline method enable both enlarge the width of Interference fringes and increase the height ambiguity so that precision of phase unwrapping and height inversion can be improved.

\section{EXPERIMENTAL RESULTS AND DISCUSSION}

\subsection{Land Classification Result}

A generic object-based multi-scale idea is introduced to the land classification (Corr,D.G., 2003).After the polarimetric analysis for different lands, surface scattering fraction as feature parameters to distinguish surface, forest, building and cultivated lands via multi-threshold segmentation at the first scale analysis layer. As it is sensitive to texture features exhibited in different types orchards and crops, the two features of gray level cooccurrence matrix (GLCM) homogeneity and dissimilarity are selected to distinguish orchards and crops via region-based segmentation at the following scale analysis layer. Then, merge the two layers to generate integral segmentation image. The each class is finally assigned at all the objects according to image object features within a search domain. The classification result for test area1 is shown in figure.6, the 8 classes are generated consisting of bare surface, crop, forest, building, lychee, mango, pawpaw and areca. By the ground data validation, the overall accuracy is up to 0.85 .
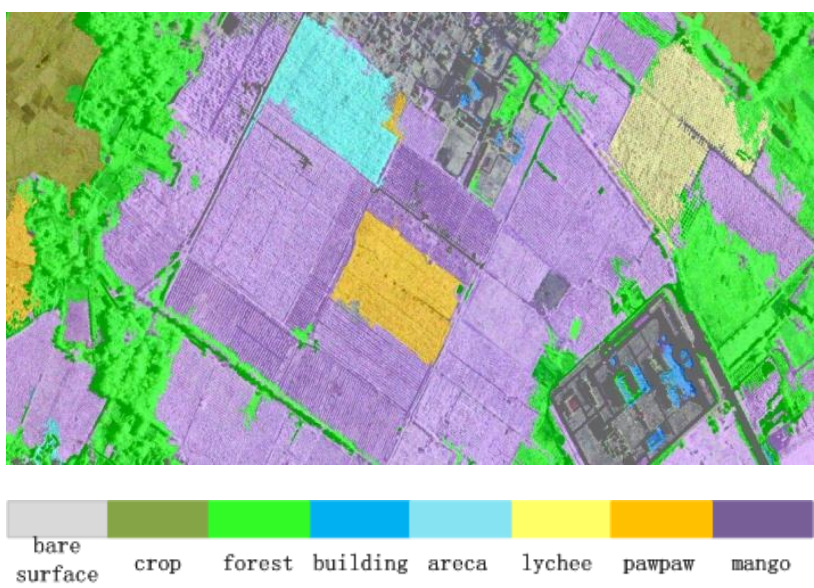

Figure.6. Land classification result of test area 1.

\subsection{Man-made Objects Detection Result}

As the Polarimetric Analysis section described, surface scattering fraction, entropy, anisotropy are all proper polarimetric descriptors for man-made objects detection. Support Vector Machine (SVM) method (B. E. Boser, 1992) is adopted to fulfil man-made objects detection with these polarimetric descriptors. Since man-made targets are the main object concerned, it is two classes of man-made objects and other objects problem research of SVM method. The SVM construct two hyperplanes that separate respectively one class and the other class. The Libsvm library (C.-C.Chang and C.-J. Lin., 2011) is employed with the RBF kernel of $\sigma=0.5$ and the cost parameter equal to 1000 .

The detection result for test area 2 is shown in figure.7., the most of man-made objects, e.g. the buildings and street lamps are mostly extracted. By the ground data validation, the overall accuracy is up to 0.89 .

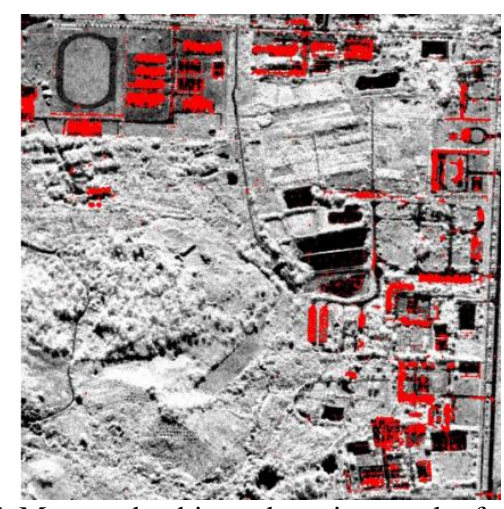

Figure.7. Man-made objects detection result of test area 2.

\subsection{DSM Mapping}

The result of ground topography mapping for the test area 3 can be derived by a dual baseline method. Due to short wavelength of $x$ band data, it is feasible to map the height of the top of the canopy rather than the height of the underlying terrain. It is more accurate to call the map digital surface model (DSM). As the Ground Control Points (GCP) for precision evaluation are not available in the test area, the DSMs derived from three different methods (MLE, ESM and the proposed method in the paper) are compared.

The coherence map with a $9 \times 9$ pixel window are calculated, three coherence maps from different height estimation methods can be compared. As shown in figure.8., coherence histograms in green, red, black colors indicates ESM method has the highest coherence depending on its own characteristics of coherence maximum, and the dual baseline method also has higher coherence than MLE method because dual baseline method implements coherence optimization processing whereas MLE method not.

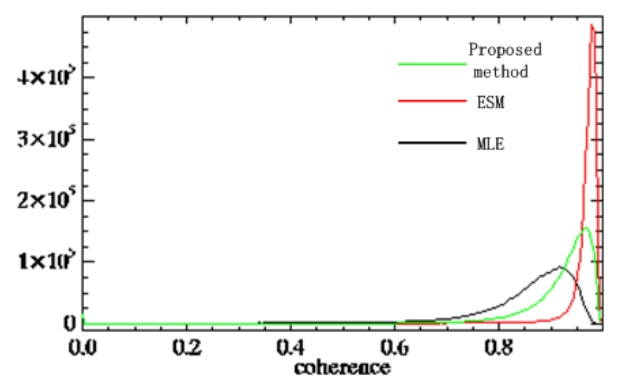

Figure.8.Coherence histograms of three methods

DSMs derived from three methods can be obtained shown in figure.9. As shown in figure.10., histograms in green, red, white colors show the height distribution of three methods. Although accurate GCPs are not available, the range of elevation of test area 3 can be searched about 20 35 meter. We can find in the green histogram that the height distribution of dual baseline is most approaching the true elevation of test area 3 as its peak value is close to 30 meter. Contrast with the dual baseline method, other two methods both deviate the true elevation range about 10 and 30 meters. It demonstrates dualbaseline polarimetric interferometry method has better elevation precision than MLE and ESM. 


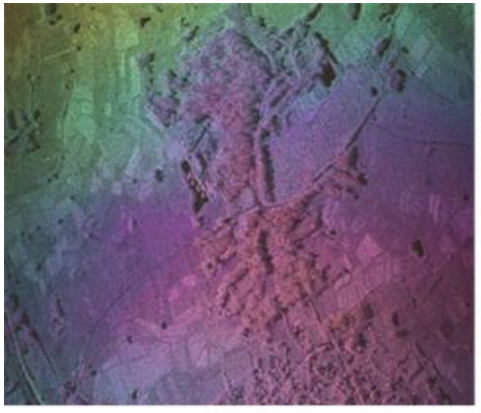

(a) MLE

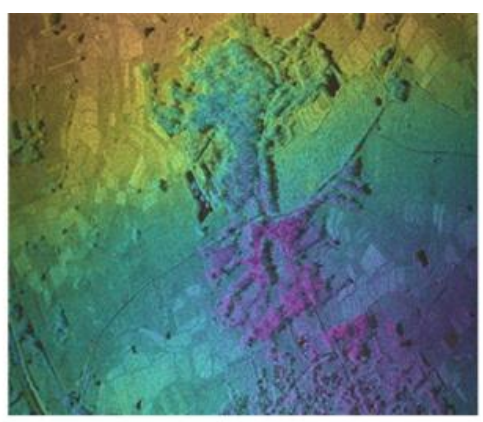

(b) ESM

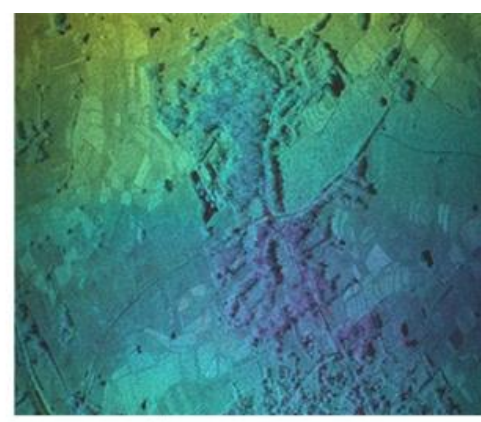

(c) Proposed method

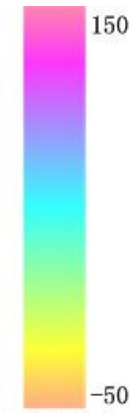

height (m)

Figure.9. DSM mapping results using three methods.

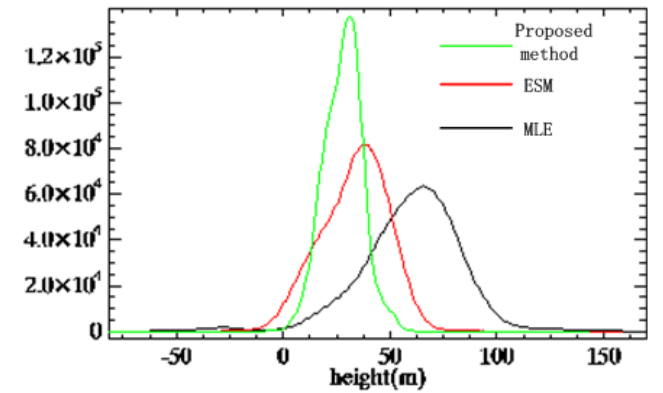

Figure.10. Surface height histograms of three methods.

\section{CONCLUSION}

The Multi-mode-XSARdataset is applicable for land classification object detectionand DSM mapping. First, polarimetric analysis has shown that X-band can provide a good discrimination between the different land types. Next, the experiments employing the selected polarimetric descriptors for land classification and man-made objects detection show the higher accuracy results. In addition, concerned with the characteristics of the Multi-mode-XSAR datasets, another experiment of DSM mapping employing the proposed dualbaseline polarimetric interferometry method has been proved to have the potentials of promotion of elevation accuracy.

\section{REFERENCES}

B. E. Boser, I. Guyon, and V. Vapnik, 1992. A training algorithm for optimal margin classifers. In Proceedings of the Fifth Annual Workshop on Computational Learning Theory, pp. 144-152.ACM Press.

C.-C. Chang and C.-J.Lin., 2011. LIBSVM -- A Library for Support Vector Machines.

http://www.csie.ntu.edu.tw/cjlin/libsvm(5 Nov, 2011)

Corr,D.G., Walker, A., Benz, U.,Lingenfelder, I., Rodrigues, A., 2003. Classification of urban SAR imagery using object oriented techniques.Proc. IGARSS, pp.198-190.

E.Colin-Koeniguer, N.Trouvé, 2010. A review about alternatives to classical Polarimetric SAR parameters. $20108^{\text {th }}$ European conference on Synthetic Aperture Radar, Germany. pp.414-417.

Elise Colin, Cecil Titin-Schnaider, and WalidTabbara, 2006.An Interferometric Coherence Optimization Method in Radar Polarimetry for High-Resolution Imagery, IEEE Trans. Geosci. Remote Sens., vol. 44, no. 1, pp. 167-175.
Jaan Praks, Elise Colin-Koeniguer, and MarttiT.Hallikainen, 2009. Alternatives to Target Entropy and Alpha Angle in SAR Polarimetry, IEEE Trans. Geosci.Remote Sens., vol.47, no7, pp.2262-2274.

S.R.Cloude and E. Pottier, 1997. Anentropy based classification scheme for land applications of polarimetricSAR,'IEEE Trans. Geosci. Remote Sens., vol.35, no.1, pp.68-78.

S. Sauer, L. Ferro-Famil, A. Reigber, and E. Pottier, 2007. Multibaseline POL-InSAR analysis of urban scenes for 3D modeling and physical feature retrieval at L-band.Proc. IGARSS, pp. 1098-1101.

\section{ACKNOWLEDGEMENTS}

The work was supported by national 863 project (Grant. No.2011AA120401). The authors would like to thank CECT-38 for providing the PolInSAR data. 